\title{
Surgical Care Improvement Project measure for postoperative glucose control should not be used as a measure of quality after cardiac surgery
}

Damien J. LaPar, MD, MSc, James M. Isbell, MD, MSCI, John A. Kern, MD, Gorav Ailawadi, MD, and Irving L. Kron, MD

Objective: The current Surgical Care Improvement Project (SCIP) measure for controlled postoperative 6-AM glycemic control after cardiac surgery identifies those with blood glucose levels of $\leq 200 \mathrm{mg} / \mathrm{dL}$. The purpose of the present study was to evaluate the effect of achieving this SCIP measure on risk-adjusted postoperative cardiac surgical outcomes.

Methods: The data were analyzed for all cardiac surgery patients from a single institution (June 2010 to August 2012). The patients were categorized by the postoperative 6-AM glucose levels into 2 SCIP measure cohorts: SCIP $(\leq 200 \mathrm{mg} / \mathrm{dL})$ versus non-SCIP $(>200 \mathrm{mg} / \mathrm{dL})$. Propensity-matched cohort comparisons and multiple regression analyses assessed the associations between SCIP measure compliance and the risk-adjusted outcomes.

Results: Of 1703 patients, $1527(90 \%)$ achieved SCIP measure glycemic control. Preoperative diabetes was more common among the non-SCIP patients $(P<.001)$; the median Society of Thoracic Surgeons-predicted mortality $(P=.14)$ was similar between the 2 groups. No significant differences were observed in major morbidity, mortality, or resource usage among the propensity-matched cohorts. After adjustment for Society of Thoracic Surgeons-predicted risk, non-SCIP status was not associated with increased mortality $(P=.44)$, composite major morbidity $(P=.16)$, major sternal complications $(P=.68)$, total intensive care unit duration $(P=.70)$, or postoperative length of stay $(P=.27)$. Similar risk-adjusted results were estimated for patients undergoing isolated coronary artery bypass grafting.

Conclusions: Achieving the SCIP measure for controlled postoperative 6-Am blood glucose levels $\leq 200 \mathrm{mg} / \mathrm{dL}$ after cardiac surgery was not associated with improved risk-adjusted mortality, morbidity, or hospital resource usage. These data suggest that this metric might not be a valid measure of postoperative cardiac surgical quality. (J Thorac Cardiovasc Surg 2014;147:1041-8)

Hyperglycemia is ubiquitous among critically ill patients and in the postoperative setting after cardiac surgery. ${ }^{1}$ Multifactorial in nature, postoperative hyperglycemia results from the concomitant effects of stress-induced release of cytokines and the influence of cardiopulmonary bypass, dextrose-containing fluids, corticosteroids, and the glucogenic effects of epinephrine and other catecholamines..$^{2-5}$

Historically, the treatment of hyperglycemia $<200$ $\mathrm{mg} / \mathrm{dL}$ in the postoperative setting was not considered the

\footnotetext{
From the Virginia Interdisciplinary CardioThoracic Outcomes Research (VICTOR) Center, Division of Thoracic and Cardiovascular Surgery, Department of Surgery, University of Virginia School of Medicine, Charlottesville, Va.

Disclosures: Authors have nothing to disclose with regard to commercial support.

Read at the 39th Annual Meeting of The Western Thoracic Surgical Association, Coeur d'Alene, Idaho, June 26-29, 2013.

Received for publication June 30, 2013; revisions received Nov 6, 2013; accepted for publication Nov 12, 2013; available ahead of print Jan 13, 2014.

Address for reprints: Irving L. Kron, MD, Virginia Interdisciplinary Cardiothoracic Outcomes Research Center, Division of Thoracic and Cardiovascular Surgery, Department of Surgery, University of Virginia School of Medicine, PO Box 800679, Charlottesville, VA 22908 (E-mail: ilk@virginia.edu). 0022-5223/\$36.00

Copyright (c) 2014 by The American Association for Thoracic Surgery http://dx.doi.org/10.1016/j.jtcvs.2013.11.028
}

standard of care for cardiac surgical patient populations. More recently, however, studies of critically ill patient populations have demonstrated a negative association between stress-induced hyperglycemia and poor outcomes. ${ }^{5-13}$ One noteworthy study of cardiac surgical patients demonstrated an increased association between sternal wound complications and elevated blood glucose (BG) levels. ${ }^{14}$ In response to that study, the Surgical Care Improvement Project (SCIP) developed a quality performance measure to maintain the 6-AM controlled blood glucose levels at $<200 \mathrm{mg} / \mathrm{dL}$ on postoperative days (PODs) 1 and 2. ${ }^{15}$ Although select single-institutional retrospective series have evaluated the current SCIP measure, most reports have focused on evaluating whether the SCIP measure is a good proxy for postoperative glycemic control, and a few have reported on unadjusted outcomes data. ${ }^{16}$ Thus, additional investigation to more firmly establish the appropriateness of the current SCIP measure within risk-adjusted cardiac surgical patient populations is needed.

The purpose of the present study was to evaluate the effect of achieving the SCIP measure for controlled BG on risk-adjusted postoperative cardiac surgical outcomes. 


$$
\begin{aligned}
& \text { Abbreviations and Acronyms } \\
& \begin{aligned}
\text { BG } & =\text { blood glucose } \\
\text { CABG } & =\text { coronary artery bypass grafting } \\
\text { ICU } & =\text { intensive care unit } \\
\text { POD } & =\text { postoperative day } \\
\text { SCIP } & =\text { Surgical Care Improvement Project } \\
\text { STS } & =\text { Society of Thoracic Surgeons }
\end{aligned}
\end{aligned}
$$

We tested the null hypothesis that the postoperative outcomes would not be significantly different after cardiac surgery as a function of SCIP measure failure.

\section{METHODS \\ Patients}

The University of Virginia institutional review board approved the present study, including an institutional waiver for the need to obtain patient consent. The data from all patients undergoing cardiac surgery at the University of Virginia were entered prospectively into a certified institutional Society of Thoracic Surgeons (STS) Adult Cardiac Surgery database. We retrospectively reviewed all adult (age $>18$ years) patients undergoing cardiac operations from June 2010 to August 2012. During the study period, our institution prospectively gathered the 6:00-AM BG levels on PODs 1 and 2, with the specific intention of evaluating our performance with the currently established SCIP measure. The patient records were stratified into 2 primary study cohorts according to the achievement of the SCIP measure: SCIP (6-AM BG $\leq 200 \mathrm{mg} / \mathrm{dL}$ ) versus non-SCIP (6-AM BG $>200 \mathrm{mg} / \mathrm{dL}$ ).

The patient demographics, preoperative risk factors, operative features, and postoperative outcomes were compared between the 2 study groups. Consistent with the current SCIP measure guideline definitions, the POD 1 and 2 6:00-AM BG values were the single serum values obtained on each postoperative morning at or near (but before) 6:00 AM. The established STS definitions were used for all preoperative variables, postoperative complications, and outcomes. ${ }^{17}$ All patient outcomes of interest were established a priori before data collection. Operative mortality was defined as patient deaths occurring before hospital discharge or within 30 days postoperatively. The composite incidence of major morbidity was defined by the incidence of permanent stroke, perioperative myocardial infarction, prolonged mechanical ventilation $(>24$ hours), pneumonia, and renal failure. Major sternal complications included the composite incidence of deep sternal wound infection, sternal dehiscence, and mediastinitis.

\section{Statistical Analysis}

The primary outcomes of interest were differences in the propensitymatched and risk-adjusted outcomes as a function of SCIP measure failure. The secondary outcomes of interest included differences within the entire study cohort with respect to the unadjusted incidence of postoperative morbidity, mortality, and resource usage between the SCIP measure groups. All study group comparisons were unpaired. Categorical variables were compared using either Pearson's $\chi^{2}$ or Fisher's exact test, and continuous variables were compared using Student's $t$ test for normally distributed data or the Wilcoxon rank sum test for non-normally distributed data, as appropriate.

To account for the potential confounding effects between the study cohorts on the postoperative outcomes, propensity score matching was performed to generate a study cohort of matched SCIP and non-SCIP patients. The propensity scores were estimated using logistic regression modeling, with the failure to achieve the SCIP measure (non-SCIP status) as the response variable and patient age, gender, operative status (elective, urgent, emergency), procedure type, and reoperation (vs primary operation) status as the possible confounding predictor variables. The propensity scores were then used to match the SCIP and non-SCIP patients in a 1:1 ratio using the nearest neighbor greedy method, resulting in equalsize study cohorts. The postoperative outcomes were then compared between the matched groups using standard univariate statistical tests of association.

To further account for potential confounding in the patient risk profiles and to verify the results observed in the propensity-matched cohorts, multivariate regression models were used to estimate the risk-adjusted associations between the predictor variable of interest (non-SCIP status) and the likelihood of several different response variables: mortality, major morbidity, major sternal complication, total intensive care unit (ICU) duration, and postoperative length of stay. All model response variables were selected a priori, and the estimated associations between non-SCIP status and outcomes were adjusted for the patient risk profiles using the established calculated STS predictive indexes, Predicted Risk of Mortality and Predicted Risk of Mortality or Morbidity, as the other modeled factors. Logistic regression model discrimination between each response variable and the predictor variables was assessed using model c-statistics. The amount of variance in the response variable explained by the model was assessed using Nagelkerke pseudo- $R^{2}$ values, and the Hosmer-Lemeshow test was used to assess model calibration across the deciles of observed and predicted risk.

All categorical variables are expressed as a percentage of the group of origin, and continuous variables are expressed as either the mean \pm standard deviation or median and interquartile range. Odds ratios with the $95 \%$ confidence intervals are used to report the results of the multivariable logistic regression analyses, and predictor variable coefficients with a standard error are reported for multivariate linear regression analyses. All reported $P$ values are 2-tailed. Data analysis was performed using $\mathrm{R}$ statistical software, version 2.12.1 (The R Foundation for Statistical Computing, available at: http://www.R-project.org).

\section{RESULTS}

\section{Comparison of Patient Characteristics and Risk Factors}

A total of 1703 patients underwent cardiac operations at the University of Virginia during the study period. Achievement of the SCIP measure occurred in 1576 patients $(89.6 \%)$ and SCIP measure failure occurred in 176 $(10.4 \%)$. The patient demographics, preoperative characteristics, and operative features as a function of SCIP measure status are listed in Table 1.

The average patient age was similar between the 2 study cohorts. Overall, men were more commonly represented in both study groups. Non-SCIP patients presented with a greater prevalence of preoperative diabetes, dyslipidemia, peripheral arterial disease, previous percutaneous intervention and coronary artery bypass grafting (CABG). Non-SCIP patients also presented with a greater burden of coronary artery disease (3-vessel disease, $46 \%$ vs $34 \%, P<.001)$. Non-SCIP patients more frequently underwent isolated CABG (CABG-only) surgery, and SCIP patients more often underwent valve procedures with or without concomitant operations. Despite these differences, the differences in preoperative risk as defined by the calculated median STS Predicted Risk of Mortality 
TABLE 1. Descriptive statistics for patient risk factors between SCIP and non-SCIP patients

\begin{tabular}{|c|c|c|c|}
\hline Factor & $\begin{array}{c}\text { SCIP } \\
(\mathbf{n}=1527)\end{array}$ & $\begin{array}{c}\text { Non-SCIP } \\
(\mathbf{n}=176)\end{array}$ & $\begin{array}{c}P \\
\text { value }\end{array}$ \\
\hline Patient age (y) & $65.1 \pm 14.7$ & $65.1 \pm 13.2$ & .66 \\
\hline Gender & & & .98 \\
\hline Female & 34.8 & 34.7 & \\
\hline Male & 65.2 & 65.3 & \\
\hline Cerebrovascular disease & 18.2 & 19.9 & .59 \\
\hline Diabetes & 30.3 & 69.3 & $<.001$ \\
\hline Dyslipidemia & 73.5 & 83.0 & .006 \\
\hline Hypertension & 76.9 & 76.7 & .93 \\
\hline Infective endocarditis & 4.5 & 1.7 & .11 \\
\hline Peripheral arterial disease & 17.0 & 25.0 & .01 \\
\hline Renal failure & 3.5 & 4.5 & .52 \\
\hline Previous CABG & 9.2 & 14.8 & .02 \\
\hline Previous PCI & 17.8 & 25.6 & .02 \\
\hline Previous valve surgery & 8.2 & 5.7 & .3 \\
\hline Atrial fibrillation & 19.4 & 17.6 & 62 \\
\hline Previous MI & 35.3 & 40.9 & .16 \\
\hline Diseased coronary arteries (n) & & & $<.001$ \\
\hline 1 & 11.4 & 6.8 & \\
\hline 2 & 16.2 & 19.3 & \\
\hline 3 & 33.7 & 45.5 & \\
\hline $\begin{array}{l}\text { Aortic insufficiency } \\
\text { (moderate to severe) }\end{array}$ & 11.5 & 5.7 & .08 \\
\hline $\begin{array}{l}\text { Mitral insufficiency } \\
\text { (moderate to severe) }\end{array}$ & 23.0 & 19.9 & .34 \\
\hline Aortic stenosis & 32.3 & 26.7 & .04 \\
\hline Mitral stenosis & 4.1 & 6.2 & .09 \\
\hline Ejection fraction $(\%)$ & $57(43-63)$ & $57(34-63)$ & .08 \\
\hline STS PROM score (\%) & $2.0(0.8-4.9)$ & $2.3(1.0-5.3)$ & .14 \\
\hline STS PROMM score (\%) & $16.6(9.9-28.7)$ & $18.2(12.3-32.4)$ & .04 \\
\hline Procedure type & & & $<.001$ \\
\hline CABG only & 29.4 & 39.2 & \\
\hline AV replacement & 18.3 & 13.6 & \\
\hline MV repair & 3.8 & 4.0 & \\
\hline MV replacement & 3.1 & 2.8 & \\
\hline AV replacement plus CABG & 7.7 & 6.2 & \\
\hline MV repair plus $\mathrm{CABG}$ & 2.2 & 2.8 & \\
\hline MV replacement plus CABG & 0.9 & 1.1 & \\
\hline AV plus MV replacement & 0.6 & 0.6 & \\
\hline Other & 34.1 & 29.5 & \\
\hline Operative status & & & .99 \\
\hline Elective & 61.2 & 61.4 & \\
\hline Urgent & 36.0 & 35.8 & \\
\hline Emergency & 2.8 & 2.8 & \\
\hline
\end{tabular}

Data presented as mean \pm standard deviation, $\%$, or median (interquartile range). $S C I P$, Surgical Care Improvement Project; $C A B G$, coronary artery bypass grafting; $P C I$, percutaneous coronary intervention; $M I$, myocardial infarction; STS, Society of Thoracic Surgeons; PROM, Predicted Risk of Mortality; PROMM, Predicted Risk of Mortality or Morbidity; $A V$, atrioventricular; $M V$, mitral valve.

was not significantly different between the 2 groups (SCIP, $2.0 \%$ vs non-SCIP, $2.3 \% ; P=.14$ ). Most cardiac operations were performed in the elective setting within both study cohorts; approximately $40 \%$ were performed in the urgent or emergency setting.

\section{Comparison of Unadjusted Postoperative Outcomes as Function of Achieving SCIP Measure}

The distribution of postoperative BG levels on POD 1 (Figure 1, $A$ ) and POD 2 (Figure 1,B) for all patients. The median PODs 1 and 2 BG level for the non-SCIP patients was 223 and $224 \mathrm{mg} / \mathrm{dL}$ and was 135 and 141 $\mathrm{mg} / \mathrm{dL}$ for the SCIP patients, respectively $(P<.001$ for both; Figure 2). Select differences in the incidence of postoperative complications and events were also observed between the unadjusted SCIP measure study groups. No significant differences in either perioperative cardiovascular or mechanical and infection-related sternal complications were observed between the 2 groups. Postoperative hospital resource usage was also similar. However, the unadjusted incidence of prolonged mechanical ventilation $(21.6 \%$ vs $10.9 \%, P<.001)$ and renal failure $(11.4 \%$ vs $5.9 \%, P=.009$ ) was greater among the non-SCIP patients. Thus, non-SCIP patients demonstrated a greater unadjusted composite incidence of major morbidity $(21.0 \%$ vs $12.6 \%$, $P=.003)$ and operative morality $(9.5 \%$ vs $3.5 \%$, $P=.002)$.

\section{Adjusted Effect of Achieving SCIP Measure on Postoperative Outcomes (Propensity-Matched Cohort)}

In an effort to reduce the influence of bias and confounding in the reported results, the patient outcomes among the SCIP and non-SCIP groups were compared between the propensity-matched cohorts (Table 2). Overall, after propensity matching, the incidence of postoperative events and major morbidity, including deep sternal wound infection and major sternal complications, was not significantly different between the matched SCIP and non-SCIP patient populations. Furthermore, the operative mortality rates and median total ICU and postoperative length of stay were not different when stratified by SCIP measure adherence or failure.

\section{Effect of SCIP Measure Failure on Risk-Adjusted Outcomes}

To further verify the adjusted results obtained between the propensity-matched patient cohorts, the risk-adjusted effect of SCIP measure failure (non-SCIP status) on the likelihood of mortality, composite major morbidity, major sternal complications, and hospital resource usage was assessed within the entire study cohort $(n=1703)$ and a subgroup of patients undergoing CABG-only surgery $(\mathrm{n}=518)$ using regression modeling (Table 3$)$. Multivariate regression analyses demonstrated that after adjustment for patient risk profile, using either the calculated Predicted Risk of Mortality (in the mortality model) or the Predicted Risk of Mortality or Morbidity (morbidity and resource usage models) as covariates in the model, 


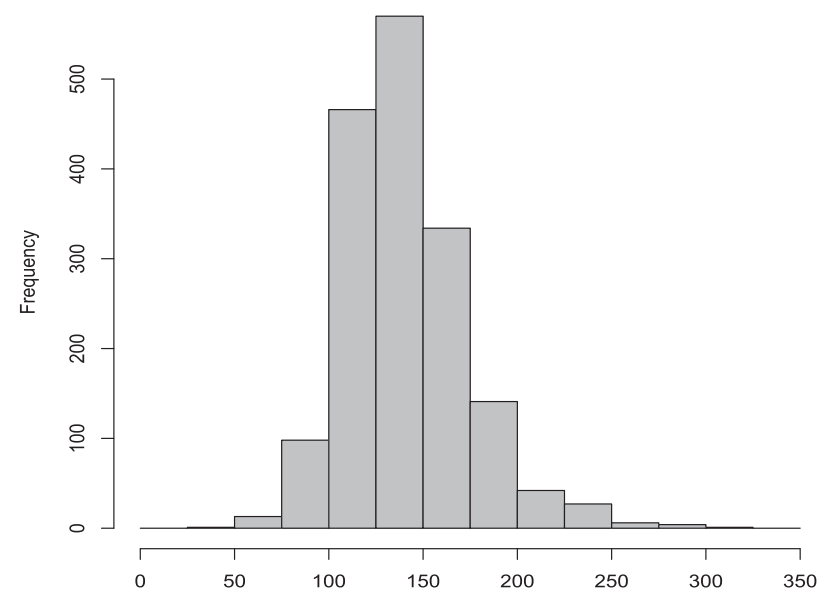

A

Postoperative Day 1 Glucose Level

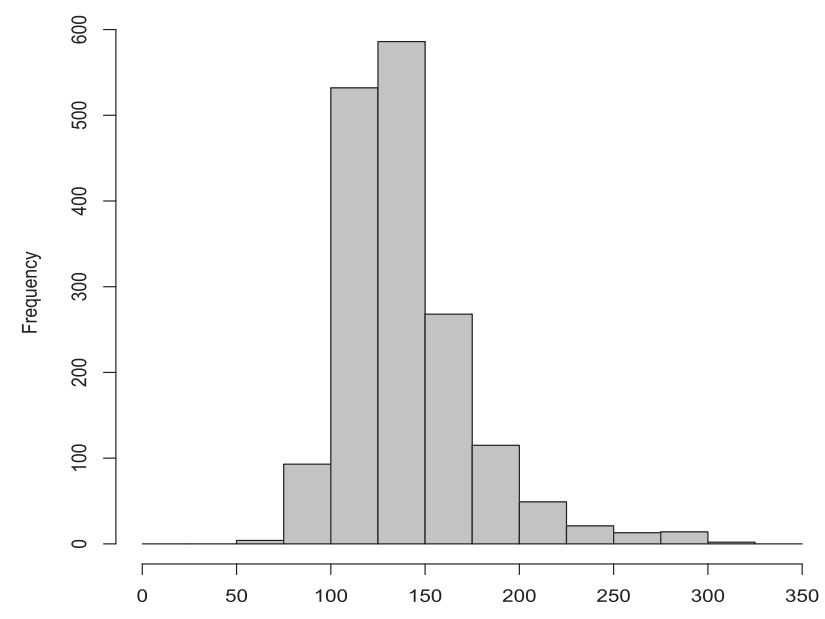

B

Postoperative Day 2 Glucose Level

FIGURE 1. Histograms displaying distribution of (A) postoperative day 1 and (B) postoperative day 2 6:00-AM blood glucose levels for all patients undergoing cardiac operations $(\mathrm{n}=1703)$.

SCIP measure failure was not associated with the probability of death $(P=.44)$, the composite incidence of major morbidity $(P=.16)$, major sternal complications $(P=.68)$, a prolonged ICU duration $(P=.69)$, or an increased postoperative length of stay $(P=.27)$. Moreover, similar results were obtained for the CABG-only cohort. Each statistical model achieved adequate discrimination, with c-statistics ranging from 0.72 to 0.78 , and described $10 \%$ to $18 \%$ of the variance existing in each response variable (ie, Nagelkerke pseudo- $R^{2}=0.10$ $0.18)$. Model calibration for all logistic regression models was acceptable, with Hosmer-Lemeshow $P>.05$.

\section{DISCUSSION}

The present study has reported on the effect of the established SCIP quality performance measure for postoperative
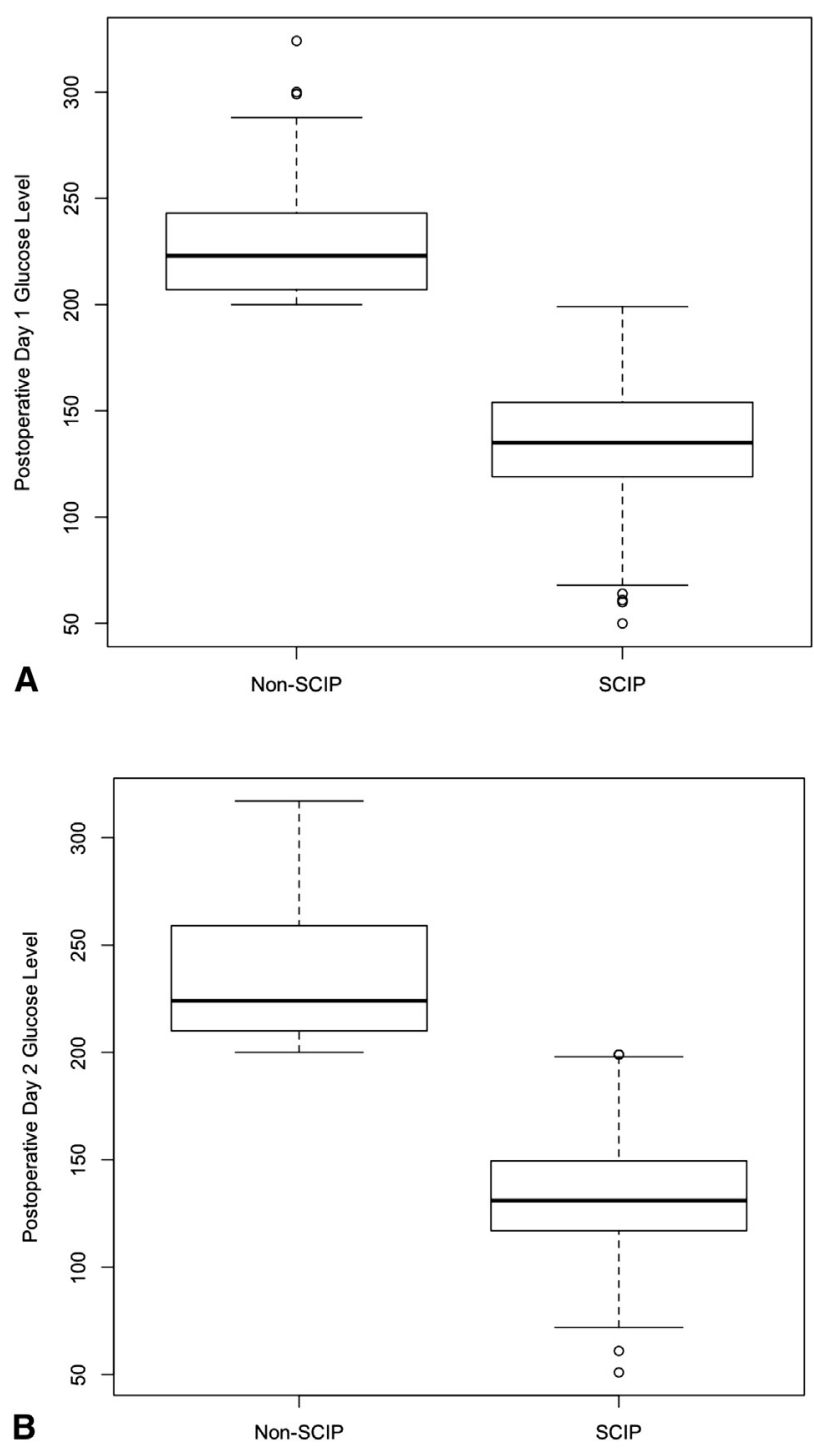

FIGURE 2. Box plots for (A) postoperative day 1 and (B) postoperative day 2 6:00-Am blood glucose levels for Surgical Care Improvement Project $(S C I P)$ versus non-Surgical Care Improvement Project (NSCIP) study cohorts $(\mathrm{n}=1703)$.

controlled BG among cardiac surgery patients. Our findings from a total study cohort of 1703 patients undergoing cardiac surgery at a single institution, suggest that achievement of the SCIP measure of controlled 6-AM BG on PODs 1 and 2 does not improve the patient outcomes in both propensitymatched cohort analyses and mortality and morbidity riskadjustment models. Furthermore, a lack of association between SCIP measure failure and patient outcomes was established within a subset of patients undergoing isolated CABG. Overall, these results have expanded previous investigative efforts that have challenged this quality measure for postoperative cardiac surgical care, and they have extended the investigation into larger patient samples with a report on the risk-adjusted outcomes. 
TABLE 2. Comparison of postoperative complications and events within propensity-matched cohorts of SCIP versus non-SCIP patients

\begin{tabular}{|c|c|c|c|}
\hline $\begin{array}{c}\text { Postoperative complication } \\
\text { or event }\end{array}$ & $\begin{array}{c}\text { SCIP } \\
(\mathbf{n}=176)\end{array}$ & $\begin{array}{c}\text { Non-SCIP } \\
(\mathbf{n}=\mathbf{1 7 6})\end{array}$ & $\begin{array}{c}P \\
\text { value }\end{array}$ \\
\hline POD 1 BG (mg/dL) & $138(120-156)$ & $223(207-243)$ & $<.001$ \\
\hline POD 2 BG (mg/dL) & $131(117-149)$ & $224(210-259)$ & $<.001$ \\
\hline Perioperative myocardial infarction & 0.0 & 0.6 & $>.99$ \\
\hline Stroke & 2.3 & 4.0 & .54 \\
\hline Pneumonia & 4.5 & 5.7 & .81 \\
\hline Prolonged ventilation & 15.9 & 21.6 & .22 \\
\hline Renal failure & 9.7 & 11.4 & .73 \\
\hline $\begin{array}{l}\text { Deep sternal wound } \\
\text { infection/mediastinitis }\end{array}$ & 0.0 & 0.6 & $>.99$ \\
\hline Major sternal complication & 0.0 & 0.6 & $>.99$ \\
\hline Composite major morbidity & 17.6 & 21 & .5 \\
\hline Operative mortality & 6.2 & 9.1 & .42 \\
\hline Total ICU length of stay (h) & $52(21-98)$ & $64(23-116)$ & .61 \\
\hline Postoperative length of stay (d) & $6(4-9)$ & $6(5-9)$ & .08 \\
\hline
\end{tabular}

Data presented as median (interquartile range) or \%. SCIP, Surgical Care Improvement Project; $P O D$, postoperative day; $B G$, blood glucose; $I C U$, intensive care unit.

Substantial research has been conducted during the past few decades to determine the effect of postoperative hyperglycemia after cardiac surgery and, perhaps more importantly, to provide clinical direction regarding the best practices to improve patient outcomes. The available published data represent prospective and retrospective levels of evidence in both diabetic and nondiabetic patient populations, many with significant methodologic issues related to potential confounding and bias. Thus, mixed results have been reported, and the debate regarding the most efficacious strategy for postoperative BG management for cardiac surgery patient populations remains unsettled.

Accumulated evidence has supported the potential beneficial effects of tight glycemic control after cardiac surgery. ${ }^{1,18-22}$ Perhaps most important was a report from Ingels and colleagues ${ }^{23}$ of 970 high-risk cardiac surgery patients that demonstrated a long-term survival benefit for those with tight glycemic control (goal BG, 80-110 $\mathrm{mg} / \mathrm{dL}$ ) compared with those with goal BG targets of 180 to $200 \mathrm{mg} / \mathrm{dL}$. Furnary and colleagues ${ }^{18}$ demonstrated a similar mortality benefit among $3500 \mathrm{CABG}$ patients with diabetes in a report that established the "Portland Protocol" for continuous postoperative intravenous insulin infusion. The same group subsequently reported on a series of an additional 2000 patients in which they advocated the use of a 3-day average of all BG measurements (3-BG) obtained from the day of surgery through the second POD to assess perioperative glycemic control. ${ }^{19}$ They demonstrated that patients with $3-\mathrm{BG}$ values $>200 \mathrm{mg} / \mathrm{dL}$ had significantly greater in-hospital mortality, deep sternal wound infection, and hospital length of stay. ${ }^{19}$ In a more recent report by Giakoumidakis and colleagues, ${ }^{21}$ however, no significant differences in the postoperative ventilation rates, infectious complications, or ICU and hospital length of stay were detected between patients with a target BG of 120 to 160 $\mathrm{mg} / \mathrm{dL}$ and those with a target BG of 161 to $200 \mathrm{mg} / \mathrm{dL}$ during the first 24 hours postoperatively. Moreover, multiple studies have associated poor glycemic control with sternal wound infections in diabetic patients after cardiac surgery, ${ }^{14,20,24}$ resulting in the development of the current SCIP measure for postoperative morning controlled BG.

The currently established SCIP measure for postoperative controlled BG after cardiac surgery has been the focus of increasing debate within the present and other investigations. ${ }^{16,25}$ In a recent report by McDonnell and colleagues, ${ }^{16}$ the validity of the current SCIP measure as a proxy for postoperative glycemic control was investigated in a cohort of 832 patients (both diabetics and nondiabetics) undergoing $\mathrm{CABG}$, valve (repair or replacement), and CABG plus valve operations. By demonstrating a lack of association between SCIP measure compliance and unadjusted patient outcomes, the investigators suggested that the SCIP measure BG values do not legitimately proxy postoperative glycemic control. ${ }^{16}$ In contrast, a report by Murphy and colleagues ${ }^{25}$ examined compliance with the SCIP 6:00-AM BG measurement among 110 consecutive cardiac surgery patients and concluded that the measurement was a valid surrogate for overall glycemic control but that the use of an insulin infusion protocol did not guarantee overall glycemic control. The incidence of SCIP noncompliance in their series was $9 \%(n=10)$. Thus, the results of these 2 series complement those from the present series and

TABLE 3. Risk-adjusted associations between SCIP measure failure (non-SCIP status) and primary study outcomes for all patients $(\mathrm{n}=1703)$ and those undergoing CABG-only surgery $(n=518)$

\begin{tabular}{|c|c|c|c|c|}
\hline \multirow[b]{2}{*}{ Outcome } & \multicolumn{2}{|c|}{ All patients } & \multicolumn{2}{|c|}{ CABG only } \\
\hline & Estimate $*(95 \%$ CI or SE) & $P$ value & Estimate $^{*}(95 \%$ CI or SE) & $P$ value \\
\hline Mortality $\dagger$ & $1.49(0.54-4.09)$ & .44 & $1.79(0.44-7.32)$ & .42 \\
\hline Major morbidity $\ddagger$ & $1.51(0.86-2.67)$ & .16 & $1.08(0.41-2.83)$ & .88 \\
\hline Major sternal complication $\ddagger$ & $1.58(0.18-13.7)$ & .68 & - & - \\
\hline Total ICU LOS (h) & $-6.93(17.8)$ & .69 & $-14.8(16.7)$ & .38 \\
\hline Postoperative LOS (d) & $0.64(0.58)$ & .27 & $-0.1(0.68)$ & .99 \\
\hline
\end{tabular}

SCIP, Surgical Care Improvement Project; $C A B G$, coronary artery bypass grafting; $C I$, confidence interval; $S E$, standard error; $I C U$, intensive care unit; $L O S$, length of stay. *Adjusted odds ratio or regression coefficient with corresponding $95 \% \mathrm{CI}$. ††Each model was adjusted for the influence of baseline patient operative risk through inclusion of †STS PROM $(P<.001$ for both models $)$ or $\ddagger$ STS PROMM $(P<.01$ for both models $)$ as other modeled factors with the regression models. 
provide a foundation for the added analyses of our report. The most significant limitations of these 2 former series concern the heterogeneity and/or small sample sizes in their analyses and a lack of adjustment for potential confounding and bias in their results. Therefore, the results of the present study have addressed these limitations in a larger patient population and have extended the analyses to control for potential confounding using propensity-matched cohort comparisons and risk-adjustment regression modeling. In the present series, the rate of SCIP measure failure $(10 \%)$ was similar to those observed in the former series, and the failure of an association between SCIP measure compliance and adjusted mortality, morbidity, and resource usage was recapitulated. Perhaps most significant was the lack of relationship between major sternal wound complications, including deep sternal wound infections and mediastinitis, and SCIP measure compliance in both unadjusted and adjusted analyses. These results support those of the previously published series by McDonnell and colleagues ${ }^{16}$ and other investigators that have demonstrated a lack of an association between SCIP measure compliance and the postoperative infection rates. ${ }^{26-28}$

In the present analyses, the outcomes related to SCIP measure compliance were also evaluated for a subgroup of patients undergoing isolated CABG, because many other series that investigated the management of postoperative hyperglycemia have restricted their analyses to CABG patients alone. ${ }^{18,19,22,29-31}$ In this subgroup of patients, SCIP measure failure also did not have a significant association with patient outcomes and resource usage after adjustment for preoperative risk using the established, and widely accepted, calculated STS predictive risk indexes. These results further strengthened the argument for reevaluation of the currently accepted SCIP measure, because CABG patients typically represent a cohort of patients with the lowest overall operative risk profiles.

The results of the present study have important clinical and patient quality-based implications, and they highlight important limitations of the current SCIP measure. Of foremost importance is the implication that the currently established SCIP measure should not be used as a valid measure of postoperative cardiac surgical quality. Second, the present results argue that the currently measured SCIP threshold value for serum BG $(200 \mathrm{mg} / \mathrm{dL})$ does not appear to adequately correlate with postoperative infection rates, other measure of morbidity, or mortality. This observation is not surprising, because most of the cited published series have investigated much narrower glycemic control ranges and suggested that patient outcomes could be improved with lower, more moderate glucose levels. Thus, the preponderance of evidence from our study and elsewhere suggests that an inherent limitation of the current SCIP measure is the failure to identify those for whom improved outcomes and surgical quality might be achieved. One possible explanation for this observation is that the current threshold of $200 \mathrm{mg} / \mathrm{dL}$ used by the SCIP measure is too high and that the category of patients with $\mathrm{BG}<200 \mathrm{mg} / \mathrm{dL}$ includes many patients who experience poor outcomes owing to relatively uncontrolled hyperglycemia (eg, BG of 180-200 $\mathrm{mg} / \mathrm{dL}$ ). Thus, the outcomes for this patient cohort might have been "inflated" and biased the results of the comparisons between the SCIP compliant and noncompliant groups, just as in the present study, toward the null. An additional limitation of the SCIP measure concerns the methods required to measure and report glycemic control. As reported in other series, measurement of a single BG level, instead of alternative measurements (eg, average glucose levels, 3-BG), might over- or underestimate the relationships between glycemic control and outcomes. These findings, therefore, add to the existing debate and call for a definitive, multi-institutional randomized clinical trial to help direct surgeons and quality assessment groups regarding the appropriate clinical standards and guidelines. In the current surgical era with an increasingly complex and higher risk patient population, emphasis on the establishment of such surgeon-directed quality performance measures remains critical.

Consistent with existing published data, our institutional practice has strongly favored aggressive postoperative glucose control. Just as demonstrated in recent analyses, the current SCIP guideline provides little guidance to surgeons, hospitals, quality performance bodies, or multidisciplinary care teams to assist in directing patient care and improving patient outcomes. Despite this, however, substantial retrospective and prospective, randomized data exist, supporting the beneficial effects of improved glycemic control on postoperative cardiac surgical outcomes and resource usage. Thus, in accordance with the latest guidelines, our institution has striven for moderate glycemic control in the perioperative setting for cardiac surgical patients, with the initiation of an established insulin infusion protocol titrated to achieve a BG level $<150 \mathrm{mg} / \mathrm{dL}$ (absolutely $<180 \mathrm{mg} / \mathrm{dL}$ ) and to maintain hypoglycemia (BG $\leq$ $70 \mathrm{mg} / \mathrm{dL}$ ) rates of $<1 \%$. We have favored the initiation of glycemic control in the operating room, the use of protocol compliance and direction measures such as computerbased protocol decision programs, and frequent (ie, hourly) point of care glucose testing in the initial postoperative period until the glucose levels have stabilized. Strict measures within our protocol for the cessation of enteral and/ or parenteral glucose sources are critical to prevent serious hypoglycemic events. We have favored alternative measures of glycemic control to those advocated by the existing SCIP measure, including daily and 3-BG or median BG measurements, in addition to tracking the percentage of glucose measurement values outside the protocol's target range and glucose variability with protocol use. Finally, we believe that a multidisciplinary quality assurance group 
charged with continuously evaluating patient compliance, performance, and outcomes remains critical to advancing protocol efforts designed to achieve improved postoperative glycemic control.

The reported results had select limitations. First, inherent selection bias should be considered in any retrospective review. Second, the analyzed 6:00-AM BG values represented single serum values obtained on the morning of PODs 1 and 2 and did not take into account the influence of BG variability occurring during the early postoperative period. However, this limitation highlights a fundamental failure of the existing SCIP measure. The reported results were limited to a single institution experience and might not be generalizable to other centers and clinical settings. In addition, the reported results were subject to the limitations of the relatively small sample sizes in our propensitymatched cohort comparisons and that our risk-adjusted models explain $10 \%$ to $18 \%$ of the variance in the modeled response variables. Thus, larger cohort analyses in the future will add increased statistical power to the present results. Finally, these analyses were restricted to the shortterm outcomes and could not provide perspective on the long-term outcomes or study endpoints. Nevertheless, the strengths of using several methods to account for potential cofounding and bias in our results have provided the largest and most contemporary analysis of risk-adjusted outcomes as a function of the current SCIP measure for cardiac surgical quality in the United States.

\section{CONCLUSIONS}

From the present results, achieving the SCIP measure for controlled postoperative 6:00-AM BG levels of $\leq 200 \mathrm{mg} / \mathrm{dL}$ after cardiac surgery was not associated with improved riskadjusted mortality, morbidity, or ICU and postoperative lengths of stay. These data suggest that this metric might not be a valid measure of postoperative cardiac surgical quality. Although the reported data did not support the current use of glycemic control as measured according to the SCIP guidelines to improve patient outcomes, our institution has strongly favored an insulin infusion-based, protocol-driven approach to postoperative glycemic control to achieve moderate target BG levels. Future prospective, multi-institution clinical trials are needed to provide more definitive clinical standards and guidelines for the management of postoperative hyperglycemia after cardiac surgery. Cardiac surgical quality standards should be determined from the highest level of clinical evidence, and surgeon involvement in the development of future quality metrics remains critical.

\section{References}

1. van den Berghe G, Wouters P, Weekers F, Verwaest C, Bruyninckx F, Schetz M, et al. Intensive insulin therapy in critically ill patients. N Engl J Med. 2001;345: 1359-67.
2. Dungan KM, Braithwaite SS, Preiser JC. Stress hyperglycaemia. Lancet. 2009; 373:1798-807.

3. Kavanagh BP, McCowen KC. Clinical practice: glycemic control in the ICU. N Engl J Med. 2010;363:2540-6.

4. Downing SW, Edmunds LH Jr. Release of vasoactive substances during cardiopulmonary bypass. Ann Thorac Surg. 1992;54:1236-43.

5. Gearhart MM, Parbhoo SK. Hyperglycemia in the critically ill patient. AACN Clin Issues. 2006;17:50-5.

6. Mizock BA. Alterations in carbohydrate metabolism during stress: a review of the literature. Am J Med. 1995;98:75-84.

7. Christiansen C, Toft P, Jorgensen HS, Andersen SK, Tonnesen E. Hyperglycaemia and mortality in critically ill patients: a prospective study. Intensive Care Med. 2004;30:1685-8.

8. Finney SJ, Zekveld C, Elia A, Evans TW. Glucose control and mortality in critically ill patients. JAMA. 2003;290:2041-7.

9. Fogelholm R, Murros K, Rissanen A, Avikainen S. Admission blood glucose and short term survival in primary intracerebral haemorrhage: a population based study. J Neurol Neurosurg Psychiatry. 2005; 76:349-53.

10. Jones KW, Cain AS, Mitchell JH, Millar RC, Rimmasch HL, French TK, et al Hyperglycemia predicts mortality after CABG: postoperative hyperglycemia predicts dramatic increases in mortality after coronary artery bypass graft surgery. J Diabetes Complications. 2008;22:365-70.

11. Laird AM, Miller PR, Kilgo PD, Meredith JW, Chang MC. Relationship of early hyperglycemia to mortality in trauma patients. J Trauma. 2004;56: $1058-62$.

12. Ouattara A, Lecomte P, Le Manach Y, Landi M, Jacqueminet S, Platonov I, et al Poor intraoperative blood glucose control is associated with a worsened hospital outcome after cardiac surgery in diabetic patients. Anesthesiology. 2005;103: 687-94.

13. Suleiman M, Hammerman H, Boulos M, Kapeliovich MR, Suleiman A Agmon Y, et al. Fasting glucose is an important independent risk factor for 30 day mortality in patients with acute myocardial infarction: a prospective study Circulation. 2005;111:754-60.

14. Zerr KJ, Furnary AP, Grunkemeier GL, Bookin S, Kanhere V, Starr A. Glucose control lowers the risk of wound infection in diabetics after open heart operations. Ann Thorac Surg. 1997;63:356-61.

15. Centers for Medicare and Medicaid Services, Joint Commission. Surgical care improvement project: percent of cardiac surgery patients with controlled 6 A.M. postoperative blood glucose. Available at: http://qualitymeasures.ahrq. gov/content.aspx?id=35532. Accessed January 1, 2013.

16. McDonnell ME, Alexanian SM, Junqueira A, Cabral H, Lazar HL. Relevance of the Surgical Care Improvement Project on glycemic control in patients undergoing cardiac surgery who receive continuous insulin infusions. J Thorac Cardiovasc Surg. 2013;145:590-4

17. Ngaage DL. Off-pump coronary artery bypass grafting: the myth, the logic and the science. Eur J Cardiothorac Surg. 2003;24:557-70.

18. Furnary AP, Gao G, Grunkemeier GL, Wu Y, Zerr KJ, Bookin SO, et al. Continuous insulin infusion reduces mortality in patients with diabetes undergoing coronary artery bypass grafting. J Thorac Cardiovasc Surg. 2003;125: $1007-21$.

19. Furnary AP, Wu Y, Bookin SO. Effect of hyperglycemia and continuous intravenous insulin infusions on outcomes of cardiac surgical procedures: the Portland Diabetic Project. Endocr Pract. 2004;10(Suppl 2):21-33.

20. Furnary AP, Zerr KJ, Grunkemeier GL, Starr A. Continuous intravenous insulin infusion reduces the incidence of deep sternal wound infection in diabetic patients after cardiac surgical procedures. Ann Thorac Surg. 1999;67:352-60; discussion 360-2.

21. Giakoumidakis K, Eltheni R, Patelarou E, Theologou S, Patris V, Michopanou N, et al. Effects of intensive glycemic control on outcomes of cardiac surgery. Heart Lung. 2013;42:146-51.

22. Lazar HL, Chipkin SR, Fitzgerald CA, Bao Y, Cabral H, Apstein CS. Tight glycemic control in diabetic coronary artery bypass graft patients improves perioperative outcomes and decreases recurrent ischemic events. Circulation. 2004 109:1497-502.

23. Ingels C, Debaveye Y, Milants I, Buelens E, Peeraer A, Devriendt Y, et al. Strict blood glucose control with insulin during intensive care after cardiac surgery: impact on 4-years survival, dependency on medical care, and quality-of-life. Eur Heart J. 2006;27:2716-24.

24. Hruska LA, Smith JM, Hendy MP, Fritz VL, McAdams S. Continuous insulin infusion reduces infectious complications in diabetics following coronary surgery. J Card Surg. 2005;20:403-7. 
25. Murphy MA, Whitman I, Campfield A, Moxey E, Haddad M, Whitman G. Intense implementation of a strict insulin infusion protocol does not guarantee postoperative glycemic control. J Am Coll Surg. 2010;211:465-9.e3.

26. Hawn MT, Itani KM, Gray SH, Vick CC, Henderson W, Houston TK. Association of timely administration of prophylactic antibiotics for major surgical procedures and surgical site infection. J Am Coll Surg. 2008;206:814-9; discussion 819-21.

27. Nguyen N, Yegiyants S, Kaloostian C, Abbas MA, Difronzo LA. The Surgical Care Improvement project (SCIP) initiative to reduce infection in elective colorectal surgery: which performance measures affect outcome? Am Surg. 2008;74:1012-6.

28. Stulberg JJ, Delaney CP, Neuhauser DV, Aron DC, Fu P, Koroukian SM. Adherence to surgical care improvement project measures and the association with postoperative infections. JAMA. 2010;303:2479-85
29. Butterworth J, Wagenknecht LE, Legault C, Zaccaro DJ, Kon ND, Hammon JW Jr, et al. Attempted control of hyperglycemia during cardiopulmonary bypass fails to improve neurologic or neurobehavioral outcomes in patients without diabetes mellitus undergoing coronary artery bypass grafting. J Thorac Cardiovasc Surg. 2005;130:1319.

30. Society of Thoracic Surgeons. Quality Performance Measures-Adult Cardiac Surgery. Available at: http://www.sts.org/quality-research-patient-safety/ quality/quality-performance-measures. Accessed January 3, 2013.

31. Desai SP, Henry LL, Holmes SD, Hunt SL, Martin CT, Hebsur S, et al. Strict versus liberal target range for perioperative glucose in patients undergoing coronary artery bypass grafting: a prospective randomized controlled trial. $J$ Thorac Cardiovasc Surg. 2012;143:318-25 